\title{
Pubis bone osteomyelitys after robotic radical cystectomy with continent intracorporeal urinary diversion: Multidisciplinary approach to a complex situation
}

\author{
Daniele Romagnoli ${ }^{1}$, Federico Mineo Bianchi ${ }^{2}$, Paolo Sadini ${ }^{2}$, Andrea Angiolini ${ }^{2}$, Daniele D'Agostino ${ }^{1}$, \\ Marco Giampaoli ${ }^{1}$, Sergio Candiotto ${ }^{3}$, Riccardo Schiavina ${ }^{2}$, Eugenio Brunocilla ${ }^{2}$, Angelo Porreca ${ }^{1}$ \\ ${ }^{1}$ Robotic Urology and Mini Invasive Urologic Surgery Unit, Abano Terme Hospital, Abano Terme (PD), Italy; \\ ${ }^{2}$ Urology Unit, S. Orsola Malpighi University Hospital - Alma Mater Studiorum, Bologna (BO), Italy; \\ ${ }^{3}$ Orthopaedic Unit, Abano Terme Hospital, Abano Terme (PD), Italy.
}

\begin{abstract}
Summary Pubic bone osteomyelitis is a rare infectious condition which is characterized by a complex diagnostic and therapeutic workup, due to its various clinical manifestations. Among the many causes of this condition, urinary fistula is the most common in case of previous urological procedures. In order to solve this complication, it is crucial to treat both the fistula and (moreover) the infectious locus arising from it, because treating the fistula alone does not provide any control on the infectious noxa. We present the first case of pubic bone osteomyelitis arising from a urinary fistula after a robotic radical cystectomy with intra corporeal continent neobladder, which has been successfully treated through a multidisciplinary approach.
\end{abstract}

KEY WORDS: Osteomyelitis; Cystectomy; Urinary fistula; Robotic; Multidisciplinary.

Submitted 28 December 2018; Accepted 8 February 2019

\section{INTRODUCTION}

Pubic bone osteomyelitis (PBO) is a rare complication that may occur after either radical prostatectomy, radical radiotherapy of the prostatic bed or other ablative techniques for the treatment of prostate cancer or benign hyperplasia (1-4). Due to its extremely low incidence, its initial cause is still uncertain. This clinical syndrome is characterized by pelvic and thigh pain, difficulty with ambulation, and recurrent urinary tract and pelvic infection. The pain associated with this condition can significantly impact the quality of life of patients and impede participation in the activities of daily living.

Several authors reported urinary fistulation (UF) into the bone symphysis to be the main culprit of this condition (5-7).

Common presentation includes chronic and debilitating suprapubic pain, fever, voiding disorders, recurrent urinary tract infections and sometimes impairment and pain during ambulation.

Our aim is to present a case of $\mathrm{PBO}$ which arose from a urinary fistula after robotic assisted radical cystectomy with continent urinary diversion, and how we managed it in order to solve the chronic inflammatory process.

\section{Case description}

We describe the case of a male patient, aged 69, who had undergone a robot-assisted radical cystectomy (RARC) with extended pelvic lymph node dissection (PLND) and intracorporeal orthotopic neobladder substitution (ONS) according to the Studer-Wiklund technique, due to a pT2 High Grade bladder carcinoma.

Local staging was performed either with CT scan and MRI in order to plan a better approach to a safe nerve sparing cystectomy, as described for prostate cancer clinical T staging (8).

The operation was performed at our institution in March 2018, and no early postoperative complication was documented. Barbed sutures were used for neo-bladder reconstruction and muscle-fascial-reconstruction as described in prostatic surgery for bladder neck-urethral anastomosis (9). Patient was discharged in $7^{\text {th }}$ post-operative day (POD), catheter was removed after 30 postoperative days, and 3 months later, during the scheduled control visit at one month, he described persistent suprapubic pain and recent presentation of $38^{\circ} \mathrm{C}$ fever. Oral empiric antibiotic therapy (prulifloxacin $600 \mathrm{mg}$ daily for seven days) was administered, but neither fever nor suprapubic pain resolved. Thus, patient was hospitalized at our Institution, to rule out a diagnostic work-up. Abdominal ultra sound (US) was negative for abdominal free fluid (suggestive for lymphocele or pelvic haematoma), and neobladder voiding was confirmed complete at ultrasound. Under the suspicion of an infection supported by neovescico-ureteric reflux (para-physiological in case of neobladder), we started intravenous antibiotic therapy (Cefotaxime $1 \mathrm{~g}$ twice a day for 7 days) and 3-way urinary catheter was inserted. Patient experienced a quick symptom relief and was discharged after 3 days. A week later catheter was removed, but after two more weeks the patient reported the recurrence of the afore mentioned symptoms. Moreover, the suprapubic pain was described as intensifying during ambulation, and was in some cases associated with mixed urinary incontinence. Patient was readmitted at our Institution, where we performed a contrast enhanced computed tomography (CT), which revealed the presence of a fili- 
form fistula between the neobladder anastomosis (at 11 o'clock) and the pubic bone. Considering the thin length $(1.5 \mathrm{~cm})$ and width $(0.5 \mathrm{~cm})$ of the fistula, and the unwillingness of the patient to be submitted to another surgical operation, conservative management was adopted. A neobladder catheter was inserted, in order to grant an adequate drainage of the neobladder, and oral empiric antibiotic therapy (Ciprofloxacin $500 \mathrm{mg}$ twice a day for 1 week every month) was administered. A follow up CT scan was performed two months later, and no residual fistula was apparently documented, thus the catheter was removed. Two weeks later a flexible neo-cystoscopy was performed, and none remnant of the fistula was documented, except for an area of edematous tissue in a location correspondent to the previous leakage.

The absence of any residual fistula was confirmed also by a subsequent retrograde uretrography. However, six months after RARC, the patient was admitted at the Emergency Department of our Institution, reporting persisting strong inguinal pain, particularly focused in the pubic symphysis. Pain was reported to have begun one month before, with a subsequent constant increase, associated with a significant impairment in the ambulation of the medial side of the right leg. Moreover, the patient reported to suffer from a significant worsening of urinary continence, using about 3 pads/day, with a steep decrease of

\section{Figure 1.}

CT scan showing fistulous tract in the context of the pubis symphysis.

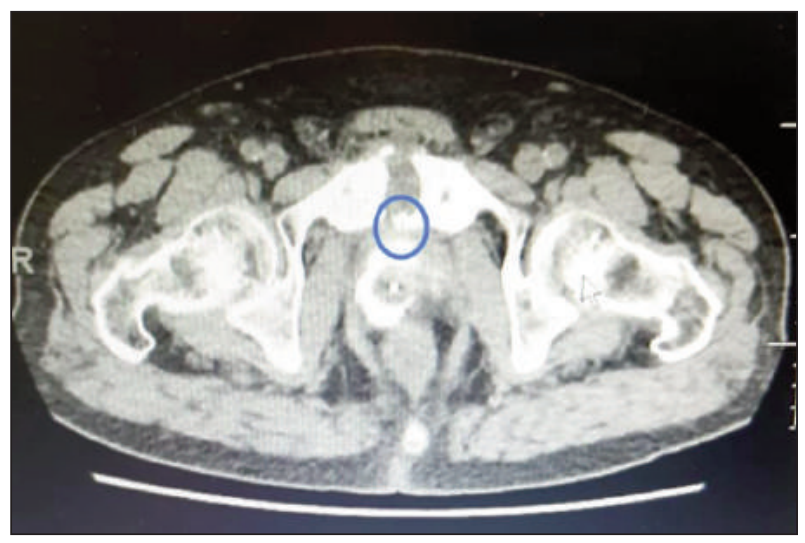

Figure 2.

Pre-operative CT urographic phase highlighting pubo-vesical fistula.

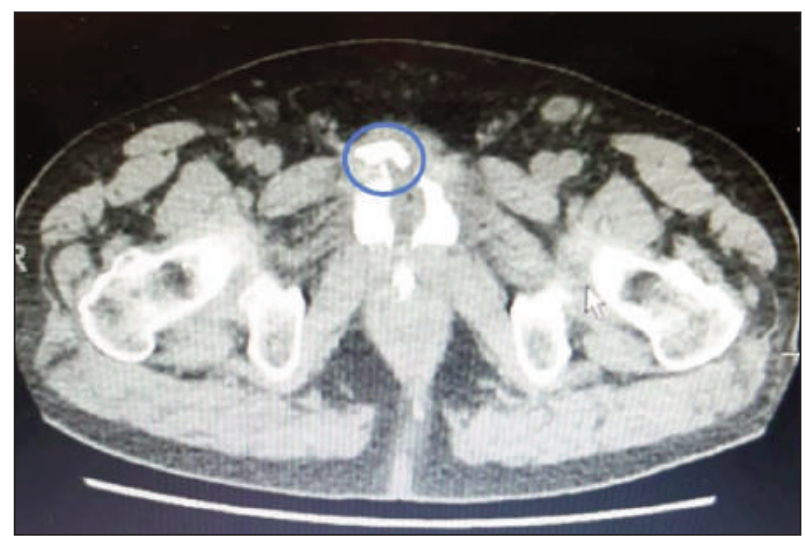

his whole quality of life. A third uro-CT was performed showing the recurrence of the fistula, which resulted to be larger than before (Figures 1-4). Physical examination confirmed intense pain at the palpation of the inguinal region, bilaterally. Due to the intense pain, the patient refused consent to the urinary catheter, so bilateral percutaneous nephrostomies were placed with combined ultrasonographic and radiologic technique, to reduce the urinary leakage from the fistula.

Multidisciplinary evaluation was carried out, by a team made up by the members of the Urology unit and Orthopedic unit. We agreed that the clinical situation was the result of a chronic pubic osteomyelitis, which had originally been started by the urinary fistula.

The recurrence of the urinary fistula appeared to be the result of the reactivation of the bone chronic inflammatory process, and the extirpation of the tissue involved was planned. Intravenous administration of meropenem $1 \mathrm{~g}$ twice a day was administered 3 days before the surgical operation, in order to reduce the septic load.

The operation began with a semicircle incision of the skin from pubis to the groin, and subsequent section of the rectus abdominis muscle. The pubic symphysis was

Figure 3.

MRI scan (T1w) showing focus of pubis osteomyelitis.

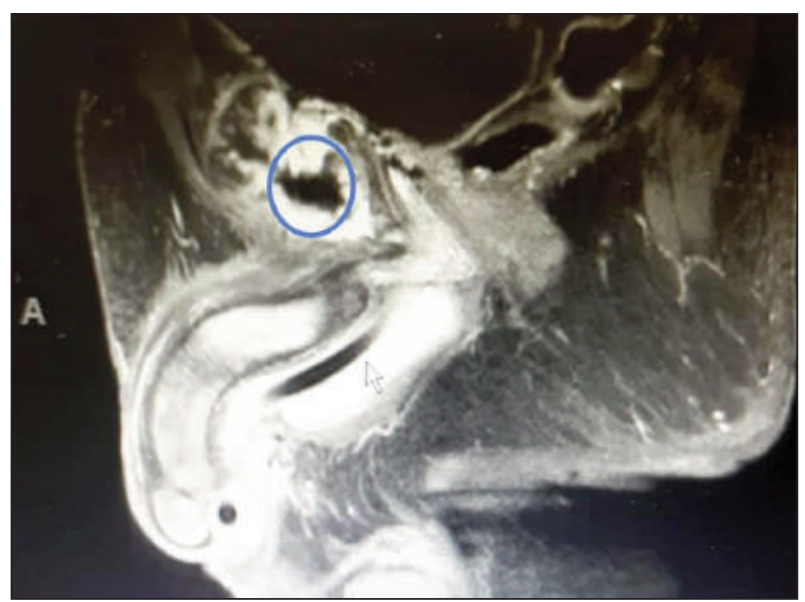

Figure 4.

MRI scan (T2w) documenting pubis osteomyelitis focus.

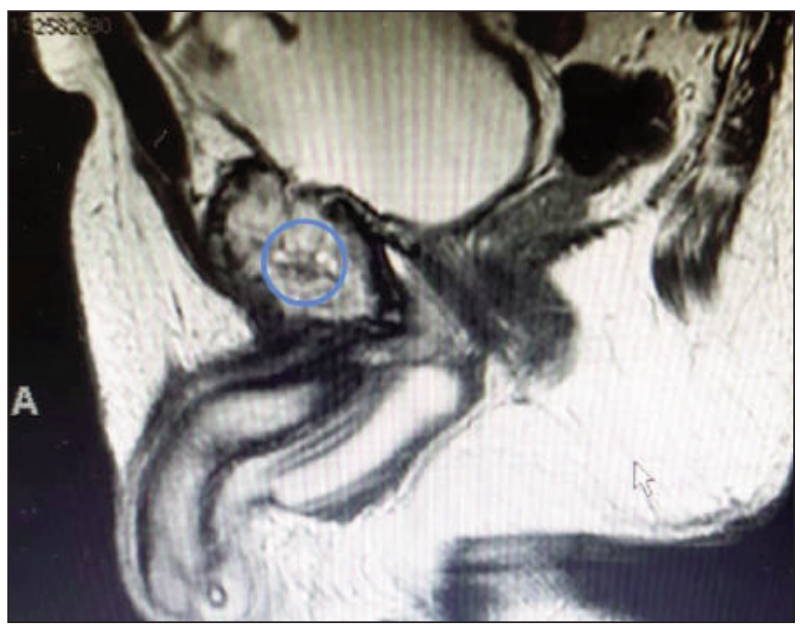


Figure 5.

Intra-operative image of the combined orthopaedic and urologic surgical procedure with removal of the osteomyelitis focus.

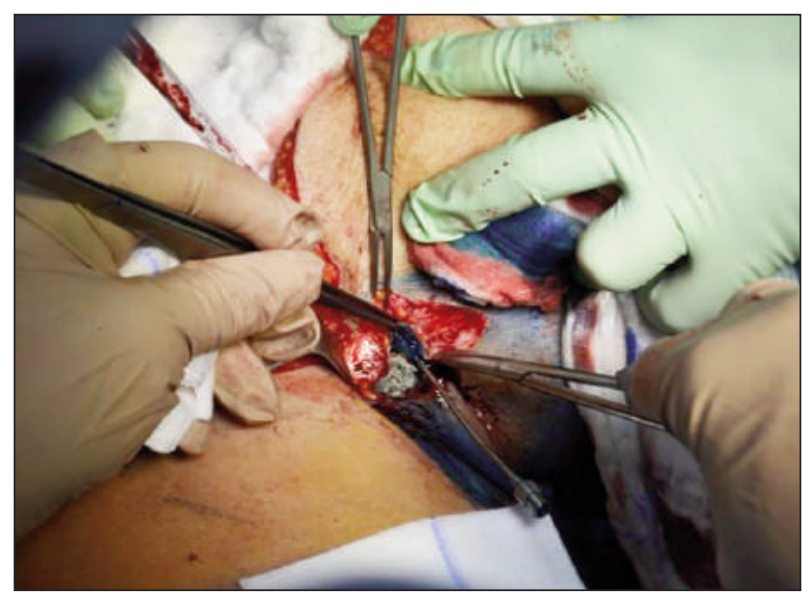

isolated, and aggressive scarring of the inflammatory tissue surrounding the fistula was performed (Figure 5). The pubic tissue was deeply removed until obtaining vital, well vascularized and bleeding tissue. Moreover, we noted the involvement of the origin of the right longus adductor muscle by the inflammatory process, thus explaining ambulation impairment. Then retrograde removal of the fistulous path was performed. Neobladder filling with a solution made up of saline and methylene blue was performed, in order to check the presence of any residual fistula. The check resulted negative, as confirmed by a flexible cystoscopy. Then the rectus abdominis muscle was fixed to the remnant part of the symphysis. Double laminar drainages were positioned at the level of the symphysis, and the surgical incision was closed. Operative time was 120 minutes, with estimated blood loss less than $100 \mathrm{~mL}$. Drainages were removed on first and third postoperative day, and length of hospital stay (LOS) was 7 days. The patient experienced a quick relief from the symptoms previously reported, also due to an adequate antalgic therapy made up by nonsteroidal-anti-inflammatory drugs (NSAIDs). One month after surgery a retrograde and anterograde radiological study was performed and resulted negative for any recurrence of the fistula. The patient reported to be fine, thus catheter was removed, and nephrostomies were closed. The patient was revaluated two weeks later and was confirmed symptoms free. Retrograde uretrography was performed resulting negative for any urinary leakage, so both nephrostomies were removed. At 3 and 6 months post-operative control visits, the patient confirmed full continence and no further symptoms.

\section{Discussion}

Osteomyelitis of the symphysis pubis is a rare entity, accounting for less than $1 \%$ of all cases of osteomyelitis (10). The presenting signs and symptoms are mainly pubic pain, antalgic gait, pain with hip motion, and occasional presence of fever. Postoperative pain after radical cystectomy is expected; nevertheless, pain lasting for more than 6 months is not usual and may suggest other etiologies $(11,12)$.

Osteomyelitis of the symphysis pubis has been reported after renal transplantation (13), inguinal herniorraphy (14), procedures for urinary stress incontinence such as tension free vaginal/transobturator taping (15), and ablative treatments for prostate cancer, such as high intensity focused ultrasound treatment for prostate cancer (16), pelvic radiation therapy and radical prostatectomy (1). Several theories lie behind the pathogenesis of PBO.

This includes infection, trauma secondary to haematogenous spread or complex regional pain syndrome (17). The most common pathogen causing pubic symphysis osteomyelitis is Staphylococcus aureus. However, other organisms such as Pseudomonas aeruginosa, Escherichia coli, Enterococcus species, Mycobacterium tuberculosis, and Salmonella species have also been reported in the literature (18). There have been several reports of pubic bone osteomyelitis secondary to pubosymphyseal fistula, in patients who underwent radical prostatectomy, followed by adjuvant radiation therapy. Matsushita et al. reported 12 patients, from two centers over an 11-year period, who developed a pubovesical fistula, following treatment of prostate cancer (19). All those patients had radiation therapy either as the primary treatment or as salvage therapy and subsequently developed bladder neck contracture. The median interval to develop pubovesical fistula was 37 months after treatment for bladder neck contracture. Broad-spectrum antibiotics were initiated in all patients, and only one patient had resolution of his osteomyelitis. The remaining patients either needed diversion of their urinary tract or insertion of bilateral percutaneous nephrostomies, to achieve resolution of symptoms. Despite such a small sample size, the longtime frame, extending over 11 years, reflects the rarity of this disease and as such the absence of any existing guidelines, for treating those fistulas. Pelvic bone pain and gait instability, after radical prostatectomy, reflect multiple bone etiologies, to contemplate in our differential diagnosis (20-22). Other than osteomyelitis of the pubic symphysis, pelvic insufficiency fracture (PIF), osteonecrosis (ON), and osteitis pubis (OP) are among the others to consider (14). OP is usually mistaken by osteomyelitis. It is defined by a painful inflammatory process resulting in bone destruction of the margins of the symphysis pubis. Nevertheless, OP is a self-limiting process, treated conservatively by anti-inflammatory drugs. The main difference between osteitis pubis and osteomyelitis is the negative culture on biopsy (7). Noteworthy here is that delay in diagnosis or treatment of symphysis pubis osteomyelitis can further manifest as bilateral thigh pain and adductor muscle abscesses, necessitating percutaneous drainage $(23,24)$. As part of pain relief, pubic bone resection has been shown to provide immediate and sustained improvement in pain, along with the long course of antibiotics administered (25). In a cohort of 16 patients, a statistically significant decrease in the median pain intensity score was noted, over a median follow-up of 9.4 months, after performing pubic bone resection ( 5.5 versus $0 ; \mathrm{p}=0.0005$ ).

Suturing of the dorsal venous complex (DVC), during the robotic radical prostatectomy procedure, using a type of 
needle called V-lock suture needle, and then fixing it at the level of the pubic bone, has been postulated to be the cause for osteomyelitis. Indeed, the use of barbed suture for urethral anastomosis in safe and efficacious but repeated needle injury to the pubic symphysis, during the urethral vesical anastomosis, may as well inadvertently cause contiguous-focus osteomyelitis (26). Yet, the possibility of an even rarer etiology of the pubic bone osteomyelitis cannot be excluded, given the negative urine culture at time of surgery.

In our experience we count only 3 cases of pubo-urinary fistula in over 15 years of high volume surgery, but the one we reported was the first after RARC ( 2 cases of UF were recorded after open radical prostatectomy). This is the first surgical complication of our series of RARC (27) whose control have been achieved through an open approach, having adopted a less invasive approach in the remaining cases (28).

In our opinion, UF and PBO have mutual cause-effect relation. $\mathrm{PBO}$ originally arose from a urinary leakage from the neo-bladder, with urinary extravasation as the main responsible for the subsequent bacterial colonization of the pubic bone. Despite long-term antibiotic therapy along with bladder catheter placement to drain urine, with further negative CT that excluded any residual urinary fistula, the patient was nonetheless readmitted in hospital 3 months later due to further recurrence of intense suprapubic pain and ambulation impairment. Despite resolution of systemic infection, antibiotic therapy could not reach and fully turn off the primary osteomyelitis focus, thus impairing tissue repairing processes and causing an additional urinary leakage.

An early diagnosis of pubic osteomyelitis is often difficult: the lack of typical acute osteomyelitis symptoms due to the deep position of pelvic bone and the limitation of its motion, along with unspecific symptoms and hematologic findings often lead to a late acknowledgement of this serious condition $(29,30)$.

X-Rays have been used for early diagnosis though appearance of bone destruction and periosteal reaction occur typically 7-14 days after onset, thus limiting their role in the early phase of disease (25).

A definitive diagnosis of pubic osteomyelitis is usually made through MRI and CT examination.

MRI displays a 94\% sensitivity and 97\% specificity in detecting this clinical condition, as it represents the most suitable imaging technique to distinguish between infected and normal bone marrow and to detect edematous changes and abscess formation. MRI should be in fact considered as PBO is suspected $(29,30)$.

The treatment for PBO is based on antibiotic therapy and surgical debridement.

When recognized early, antibiotic therapy alone could guarantee a complete resolution of this clinical condition, due to the rich vascularization of the pelvis. Del Busto et al. reviewed 7 patients diagnosed with $\mathrm{PBO}$, who didn't require any surgical debridement after complete response to 3-to-12 weeks of antibiotic therapy (31).

Surgical debridement is nonetheless recommended after primary antibiotic therapy and consists in a wide removal of necrotic and infected tissues until vital and bleeding ones are reached. Intravenous antibiotics should be prolonged for 6 more weeks after surgery, with 3 additional weeks of oral antibiotics in case of haematogenous origin of $\mathrm{PBO}(29,32)$.

In our case direct and prolonged urinary leakage from the orthotopic neobladder resulted in a chronic infection with a slowly increasing inflammatory process that could be identified only when osteomyelitis had already destroyed pubic bone and the abscess was already formed. Uro-CT and MRI imaging were decisive to correctly identifying $\mathrm{PBO}$, as symptoms reported by the patient and hematologic findings were inconclusive.

Antibiotic therapy alone was not enough to resolve PBO. Despite complete resolution of urinary fistula and apparent symptom relief, the inflammatory process recurred 2 months later. Persistence of chronic inflammation caused an impairment of tissue reparation, that translated in a recurrent urinary fistula from the neobladder.

Multi-disciplinary management was critic for choosing the proper management of this peculiar clinical condition: extensive surgical debridement of the necrotic and infected tissues along with prolonged antibiotic therapy led to a definitive resolution of the chronic inflammation. No signs of recurrence were identified at 3 and 6 months follow up visits, with a complete continence recover and any additional symptoms were reported.

\section{Conclusions}

Osteomyelitis of the symphysis pubis is a rare condition, quite uncommon to occur after radical cystectomy. It can be often missed due to vague non-specific symptoms. Its clinical presentation might be confused by the presence of concurrent urinary fistula, which might be its origin, but also its effect. In case of urinary fistula, it is very important to rule out, via MRI, the presence of any concurrent $\mathrm{PBO}$ focus, because curative goal might be achieved only if both conditions are treated, preferably through a multidisciplinary approach.

\section{REFEREMCES}

1. Gupta S. et al., Pubic symphysis osteomyelitis in the prostate cancer survivor: clinical presentation, evaluation, and management. Urology, 2015; 85:684.

2. Moore DC, et al. A 57-year-old man with a history of prostatectomy and pelvic irradiation presents with recurrent urinary tract infections, hematuria, and pelvic pain. Urology. 2013; 81:221.

3. Kats E, et al. Diagnosis and treatment of osteitis pubis caused by a prostate-symphysis fistula: a rare complication after transurethral resection of the prostate. Br J Urol. 1998; 81:927.

4. Hutchinson RC, et al. Magnetic resonance imaging to detect vesico-symphyseal fistula following robotic prostatectomy. Int Braz J Urol. 2013; 1:288.

5. Bugeja S, et al. Fistulation into the Pubic Symphysis after Treatment of Prostate Cancer: An Important and Surgically Correctable Complication. J Urol, 2016; 195:391.

6. Matsushita K, et al. Pubovesical fistula: a rare complication after treatment of prostate cancer. Urology, 2012; 80: 46.

7. Knoeller, et al. Osteitis or osteomyelitis of the pubis? A diagnostic 
and therapeutic challenge: report of 9 cases and review of the literature, Acta Orthop Belg, 2006; 72:541.

8. Schiavina R, et al. MRI Displays the Prostatic Cancer Anatomy and Improves the Bundles Management Before Robot-Assisted Radical Prostatectomy. J Endourol. 2018; 32:315.

9. Porreca A, et al. Bidirectional barbed suture for posterior musculofascial reconstruction and knotless vesicourethral anastomosis during robot-assisted radical prostatectomy, Minerva Urol Nefrol. 2018; 70:319

10. Lavien G, et al, Pubic Bone Resection Provides Objective Pain Control in the Prostate Cancer Survivor with Pubic Bone Osteomyelitis with an Associated Urinary Tract to Pubic Symphysis Fistula, Reconstructive Urology. 2017; 100:234.

11. McHenry MC, Alfidi RJ, Wilde AH, Hawk WA. Hematogenous osteomyelitis: A changing disease, Cleve Clin Q. 1975; 42:125-53.

12. Moore DC1, Keegan KA, Resnick MJ, et al. A 57-year-old man with a history of prostatectomy and pelvic irradiation presents with recurrent urinary tract infections, hematuria, and pelvic pain, Urology. 2013; 81:221.

13. Jindal RM, Idelson B, Bernard D, Cho SI.R. M. Osteomyelitis of symphysis pubis following renal transplantation, Postgrad Med J. 1993; 69:742.

14. Mader R, Yeromenco E. Pseudomonas osteomyelitis of the symphysis pubis after inguinal hernia repair, Clini Rheumatol. 1999; 18:167.

15. Goldberg RP, Tchetgen MB, Sand PK, et al. Incidence of pubic osteomyelitis after bladder neck suspension using bone anchors, Urology, 2004; 63:704.

16. Robison CM, Gor RA, Metro MJ. Pubic bone osteomyelitis after salvage high-intensity focused ultrasound for prostate cancer. Curr Urol. 2013; 7:149-51.

17. Sexton DJ, Heskestad L, Lambeth WR, et al. Postoperative pubic osteomyelitis misdiagnosed as osteitis pubis: report of four cases and review. Clin Infect Dis. 1993; 17:695-700.

18. Alqahtani SM, Jiang F, Barimani B, Gdalevitch M. Symphysis pubis osteomyelitis with bilateral adductor muscles abscess. Case Rep Orthop. 2014; 2014:982171.

19. Matsushita K, Ginsburg L, Mian BM, et al. Pubovesical fistula:
A rare complication after treatment of prostate cancer. Urology, 2012; 80:446.

20. Pauli S, Willemsen P, Declerck K, et al. Osteomyelitis pubis versus osteitis pubis: a case presentation and review of the literature. $\mathrm{Br}$ J Sports Med. 2002; 36:71-3.

21. Trubiano JA, Yang N, Mahony AA. Bilateral thigh pain after treatment for prostate cancer. BMJ Case Rep. 2013; 2013 pii: bcr2013008784.

22. Lavien G, Chery G, Zaid UB, Peterson AC. Pubic bone resection provides objective pain control in the prostate cancer survivor with pubic bone osteomyelitis with an associated urinary tract to pubic symphysis fistula. Urology. 2017; 100:234-239.

23. Kitaguchi D, et al. Pubic osteomyelitis after surgery for perforated colonic diverticulitis with fecal peritonitis: A case report. Int J Surg Case Rep. 2017; 38:50.

24. Rosenthal RE, et al. Osteomyelitis of the symphysis pubis: a separate disease from osteitis pubis. Report of three cases and review of the literature. J Bone Joint Surg Am. 1982; 64:123.

25. Kozlowski K, Hochberger O, Povysil B. Swollen ischiopubic synchondrosis: a dilemma for the radiologist. Australas. Radiol. 1995; 39:224.

26. Porreca A, et al. Robotic-Assisted Radical Prostatectomy with the Use of Barbed Sutures. Surg Technol Int. 2017; 30:39.

27. Porreca A, et al. Robot assisted radical cystectomy with totally intracorporeal urinary diversion: initial, single-surgeon's experience after a modified modular training, Minerva Urol Nefrol. 2018; 70:193.

28. Schiavina $R$, et al. Laparoscopic and robotic ureteral stenosis repair: a multi-institutional experience with a long-term follow-up. J Robot Surg. 2016; 10:323.

29. Carek PJ, et al. Diagnosis and management of osteomyelitis. Am Fam Phys. 2001; 63:2413.

30. Sammak B, et al. Osteomyelitis: a review of currently used imaging techniques. Eur. Radiol. 1999; 9:894.

31. Del Busto R, et al. Osteomyelitis of the pubis. Report of seven cases. JAMA. 1982; 248:1498.

32. Wilmes D, et al. Osteomyelitis pubis caused by Kingella kingae in an adult patient: report of the first case, BMC Infect. Dis. 2012; 12:236.

\section{Correspondence}

Daniele Romagnoli, MD (Corresponding Author)

danieleromagnoli87@gmail.com

Daniele D'Agostino, MD

Marco Giampaoli, MD

Angelo Porreca, MD

Robotic Urology and Mini Invasive Urologic Surgery Unit, Abano Terme

Hospital Piazza Cristoforo Colombo, 2 Abano Terme (PD) (Italy)

Federico Mineo Bianchi, MD

Paolo Sadini, MD

Riccardo Schiavina, MD

Eugenio Brunocilla, MD

Andrea Angiolini, MD

Urology Unit, S. Orsola Malpighi University Hospital - Alma Mater Studiorum

Via Pelagio Palagi, 9 Bologna (Italy)

Sergio Candiotto, $M D$

Orthopaedic Unit, Abano Terme Hospital

Piazza Cristoforo Colombo, 2 Abano Terme (PD) (Italy) 